\title{
Nonrelativistic hybrid geometries with gravitational gauge-fixing terms
}

\author{
Sinya Aoki, ${ }^{1}$ Janos Balog $\odot,{ }^{2}$ Shuichi Yokoyama $\odot,{ }^{1}$ and Kentaroh Yoshida ${ }^{3}$ \\ ${ }^{1}$ Center for Gravitational Physics, Yukawa Institute for Theoretical Physics, Kyoto University, \\ Kitashirakawa Oiwake-cho, Sakyo-Ku, Kyoto, Japan \\ ${ }^{2}$ Institute for Particle and Nuclear Physics, Wigner Research Centre for Physics, MTA Lendület Holographic QFT Group, \\ 1525 Budapest 114, P.O. Box 49, Hungary \\ ${ }^{3}$ Department of Physics, Kyoto University, Kitashirakawa Oiwake-cho, Sakyo-ku, Kyoto, Japan
}

(Received 16 November 2019; accepted 3 January 2020; published 19 February 2020)

\begin{abstract}
We search a gravitational system that allows a nonrelativistic hybrid geometry interpolating the Schrödinger and Lifshitz spacetimes as a solution. As such a candidate an Einstein-Maxwell-Higgs system naturally arises and we verify that this system indeed supports the hybrid geometry with the help of a gauge-fixing term for diffeomorphism. As a result, this gravitational system may be interpreted as a holographic dual of a general nonrelativistic system at the boundary.
\end{abstract}

DOI: 10.1103/PhysRevResearch.2.013169

\section{INTRODUCTION}

It is a new paradigm to realize quantum theory of gravity as a hologram of a quantum field theory at boundary [1,2] and has attracted much interest since the discovery of the AdS/CFT correspondence [3], which admits nontrivial tests by explicit computation [4,5]. So far, various kinds of holographic duals have been proposed. Among them, we are interested here in gravity duals for nonrelativistic (NR) theories based on an NR conformal symmetry called the Schrödinger symmetry [6-8] and a Lifshitz scaling symmetry [9]. (For a nice summary, see, for example, Ref. [10].)

From the point of view to construct a bulk gravitational system gradually from a boundary theory [11], it is important to specify a scale at the boundary which plays the role of the holographic radial direction. There are several proposals to describe how the bulk radial direction emerges. As one approach, some of the authors of the present letter have proposed that a holographic direction may be described by a flow equation [12-15] that coarse-grains operators in a nonlocal fashion [16,17]. In comparison to other approaches, this flow equation method has several advantages. One of them is to make it possible to construct a metric of the bulk geometry directly. In particular, it is possible to construct an AdS geometry with a general conformally flat boundary [18] or with a quantum-mechanically corrected bulk cosmological constant [19] and a holographic geometry for a general NR system [20].

The resulting metric of the holographic space-time for an NR system is expressed as a three-parameter deformation of

Published by the American Physical Society under the terms of the Creative Commons Attribution 4.0 International license. Further distribution of this work must maintain attribution to the author(s) and the published article's title, journal citation, and DOI. the $d+1$-dimensional AdS geometry [20],

$$
\begin{aligned}
d s^{2}= & \ell^{2}\left[-\alpha \frac{\left(d x^{+}\right)^{2}}{\tau^{4}}+\frac{d \tau^{2}+\left(d x^{i}\right)^{2}+2(1+\beta) d x^{+} d x^{-}}{\tau^{2}}\right. \\
& \left.+\gamma\left(d x^{-}\right)^{2}\right]
\end{aligned}
$$

where $\ell$ is the AdS radius, $i=1, \ldots, d-2$, and $\alpha, \beta, \gamma$ are real parameters satisfying

$$
\alpha \geqslant 0, \quad \alpha \gamma+(1+\beta)^{2}>0 .
$$

At a general point in the parameter space, this metric describes a $d$-dimensional Lifshitz space-time with the dynamical critical exponent 2 times a straight line, while it enhances to the Schrödinger space-time in $d+1$ dimensions when $\beta=\gamma=$ 0 . Therefore, this geometry would deserve to be called an NR hybrid geometry.

So far, while only the metric has been computed, a gravitational system to support this geometry has not been identified. In particular, the treatment of the matter sector in the context of the flow equation method has not been discussed. The purpose of this letter is to fill this gap, and to stress that the flow equation method works for the matter sector as well, not restricted to the metric. This result strongly indicates that the flow equation method can capture gravitation beyond geometry.

\section{NR HYBRID GEOMETRY FROM BOUNDARY}

\section{A. Review of the flow equation method}

In this section, we shall give a brief review of the results of Ref. [20] on how the NR hybrid geometry Eq. (1) can be derived starting from a nonrelativistic conformal field theory (NRCFT) by the flow equation method.

Let us consider a primary scalar operator $O(\vec{x}, t)$ with a general conformal dimension $\Delta$ in a $d$-1-dimensional NRCFT. Note that a primary field in NRCFT is complex 
in relation to a $\mathrm{U}(1)$ symmetry group associated with the conservation of particle number.

The two point function of primary operators is almost completely determined by an NR conformal symmetry as $\left\langle O\left(\vec{x}_{1}, t_{1}\right) O^{\dagger}\left(\vec{x}_{2}, t_{2}\right)\right\rangle=\frac{1}{\left(t_{12}\right)^{\Delta}} f\left(\frac{\vec{x}_{12}^{2}}{2 t_{12}}\right)$, where $t_{12}=t_{1}-$ $t_{2}, \vec{x}_{12}=\vec{x}_{1}-\vec{x}_{2}$, and $f(x)$ is a function depending on the given theory. The function $f$ is not necessarily an eigenfunction of the mass operator, which is the center in the nonrelativistic conformal algebra. Then, by introducing an extra direction, the mass operator is realized by a differential operator along this direction, and the two point function is now expressed as $\left\langle O\left(\vec{x}_{1}, x_{1}^{+}, x_{1}^{-}\right) O^{\dagger}\left(\vec{x}_{2}, x_{2}^{+}, x_{2}^{-}\right)\right\rangle=$ $\frac{1}{\left(x_{12}^{+}\right)^{\Delta}} f\left(x_{12}^{-}+\frac{\vec{x}_{12}^{2}}{2 x_{12}^{+}}\right)$, where $x^{-}$is the extra direction and we have set $x^{+}=t$.

To construct the holographic geometry, let us course-grain the conformal primary operator by a nonrelativistic free flow equation of the form

$$
\begin{aligned}
\frac{\partial}{\partial \eta} \phi(x ; \eta) & =\left(\vec{\partial}^{2}+2 \partial_{-} \partial_{+}+2 i \bar{m} \partial_{+}\right) \phi(x ; \eta), \\
\phi(x ; 0) & =O(x),
\end{aligned}
$$

where $\eta$ is a smearing variable and $\bar{m}$ is a real parameter of mass dimension one. Due to the parameter $\bar{m}$, the twopoint function of the flowed operator $\phi$ is free from the contact singularity in a general parametrization. Thus, the flowed field can be normalized as $\sigma(x ; \eta)=\frac{\phi(x ; \eta)}{\sqrt{\left\langle\phi(x ; \eta) \phi^{\dagger}(x ; \eta)\right\rangle}}$, so that $\left\langle\sigma(x ; \eta) \sigma^{\dagger}(x ; \eta)\right\rangle=1$. Then the two-point function of normalized fields is given by

$$
\begin{aligned}
& \left\langle\sigma\left(x_{1} ; \eta_{1}\right) \sigma^{\dagger}\left(x_{2} ; \eta_{2}\right)\right\rangle \\
& \quad=\left(\frac{4 \eta_{1} \eta_{2}}{\eta_{+}^{2}}\right)^{\Delta / 2} G\left(\frac{2\left(x_{12}^{+}+2 i \bar{m} \eta_{+}\right) x_{12}^{-}+\left(\vec{x}_{12}\right)^{2}}{\eta_{+}}, \frac{x_{12}^{+}}{\eta_{+}}\right),
\end{aligned}
$$

where $\eta_{+}=\eta_{1}+\eta_{2}$ and $G(u, v)$ is a scalar function (depending on the original $f$ ) satisfying $G(0,0)=1$.

By using the normalized field, the so-called metric operator can be defined as

$$
\hat{g}_{M N}(x ; \eta) \equiv \frac{1}{2} \partial_{\{M} \sigma(x ; \eta) \partial_{N\}} \sigma^{\dagger}(x ; \eta),
$$

where the bracket $\{$,$\} denotes symmetrizing the indices like$ $\{A, B\}:=A B+B A$.

The proposal is that the vacuum expectation value of the metric operator Eq. (4) provides the metric of the bulk space, $d s^{2}=\left\langle\hat{g}_{M N}\right\rangle d z^{M} d z^{N}$, where $z^{M}$ are the coordinates describing the bulk space. Note that the flow variable $\eta$ is related to the holographic radial coordinate. The resulting metric is given by

$$
\begin{aligned}
d s^{2}= & \frac{\Delta}{4 \eta^{2}} d \eta^{2}+\frac{-G^{(0,2)}(\overrightarrow{0})}{4 \eta^{2}}\left(d x^{+}\right)^{2}+(4 \bar{m})^{2} G^{(2,0)}(\overrightarrow{0})\left(d x^{-}\right)^{2} \\
& +2 \frac{-G^{(1,0)}(\overrightarrow{0})-2 i \bar{m} G^{(1,1)}(\overrightarrow{0})}{\eta} d x^{+} d x^{-} \\
& +\frac{-\delta_{i j} G^{(1,0)}(\overrightarrow{0})}{\eta} d x^{i} d x^{j}
\end{aligned}
$$

where $\left.G^{(n, m)}(\overrightarrow{0}) \equiv \frac{\partial^{n}}{\partial X^{n}} \frac{\partial^{m}}{\partial Y^{m}} G(X, Y)\right|_{X=Y=0}$. There are constraints coming from the flow Eq. (3) like $-\Delta=2(d-$ 1) $G^{(1,0)}(\overrightarrow{0})+8 i \bar{m} G^{(1,1)}(\overrightarrow{0})+2 i \bar{m} G^{(0,1)}(\overrightarrow{0})$. By performing a coordinate transformation $\eta=-\frac{G^{(1,0)}(\overrightarrow{0})}{\Delta} \tau^{2}$, the metric Eq. (5) can be rewritten to the form Eq. (1) under the identification $\alpha=\frac{\Delta G^{(0,2)}(\overrightarrow{0})}{4 G^{(1,0)}(\overrightarrow{0})^{2}}, \beta=\frac{2 i \bar{m} G^{(1,1)}(\overrightarrow{0})}{G^{(1,0)}(\overrightarrow{0})}, \gamma=\frac{4 \bar{m}^{2} G^{(2,0)}(\overrightarrow{0})}{\Delta}, \ell^{2}=\Delta$. Note that $\tau$ is the radial direction in AdS.

\section{B. Searching for the dual bulk theory}

It is natural to ask if there exists a gravitational theory which exhibits the NR hybrid geometry Eq. (1) as a solution to the equations of motion. To look for the dual bulk theory it is important to find out the so-called pregeometric operators, which convert to geometric objects after taking the expectation value [19]. If there exists a symmetry group such as $O(N)$ whose rank is taken to be large for the emergence of the bulk, then these operators are constructed so as to be invariant under its global transformation.

In the current situation the phase rotation group plays this role, and operators invariant under the action are not only the spin-2 operator $\hat{g}_{\mu \nu}$ but also the following spin-1 operator

$$
\hat{A}_{\mu}(x ; \eta) \equiv \frac{i}{2}\left[\partial_{\mu} \sigma(x ; \eta) \sigma^{\dagger}(x ; \eta)-\sigma(x ; \eta) \partial_{\mu} \sigma^{\dagger}(x ; \eta)\right] .
$$

Due to the fact that a nonrelativistic conformal primary field takes values in the complex numbers, the vacuum expectation value of the spin-1 operator is nontrivial: $A_{-}(z)=-4 \bar{m} G^{(1,0)}(\overrightarrow{0}) \quad$ and $\quad A_{+}(z)=-\frac{i \Delta G^{(0,1)}(\overrightarrow{0})}{2 G^{(1,0)}(\overrightarrow{0}) \tau^{2}}$, where we have set $A_{\mu}(z)=\left\langle\hat{A}_{\mu}(x ; \eta)\right\rangle$, and the other components vanish. Therefore, one can expect that not only the metric tensor $g_{\mu \nu}$ but also the gauge field $A_{\mu}$ may dynamically exist in the bulk theory.

In fact, this anticipation is consistent with a common understanding in the holography that a global symmetry of the boundary theory becomes a gauge symmetry of the bulk theory [4]. (See also Refs. [21,22].) To see this, let us gauge the $\mathrm{U}(1)$ phase rotation $O \rightarrow e^{i \lambda} O$ so that the parameter depends not only on the coordinates in the boundary but also the radial direction in the bulk. Then the spin-1 operator is transformed as $A_{\mu} \rightarrow A_{\mu}-\partial_{\mu} \lambda$, which is an abelian gauge transformation. Thus, one can see that the spin-1 operator is converted to a gauge field in the bulk. This result indicates that the symmetry associated with the particle number corresponds to the abelian gauge symmetry in the bulk [23]. Applying this argument to the flowed primary operator $\sigma$, this physical degree of freedom converts to a charged scalar field with the gauge charge unity.

Then a spin-2 operator defined in a suitable way should be invariant under the local U(1) transformation, while the one defined above is not. There is still a possibility that extra terms can be absorbed by diffeomorphism, though our preliminary analysis suggests that it may happen by considering a special situation. We leave the detailed analysis of this issue to a future work. (A possible way to avoid this problem is to represent the bulk metric by an information metric [15] $d s_{\text {inf }}^{2}:=\frac{1}{2} \operatorname{tr}(d \rho(z) d \rho(z))=g_{M N}^{\inf }(z) d z^{M} d z^{N}$, where $\rho(z)$ is the density matrix defined by $\rho(z):=\sigma(x ; \eta)|0\rangle\langle 0| \sigma(x ; \eta)^{\dagger}$, and an explicit form of $g_{M N}^{\inf }(z)$ can be found in the footnote 1 of Ref. [20]. This is invariant under the local U(1) transformation, whereas a metric operator whose vacuum expectation value becomes $g_{M N}^{\inf }(z)$ seems to be absent, unless the large 
$N$ factorization occurs, which causes a trouble, for instance, when correlation functions of gravitons are computed from the boundary by using this framework.)

As a result, one may argue that the field contents in the bulk theory are the metric, an abelian gauge field and a charged scalar field, and thus the dual bulk theory should be a low energy effective action describing the dynamics of these fields. A natural candidate of the system may be the Einstein-Hilbert action minimally coupled to an abelian gauge field as well as a charged scalar field with a certain potential. This system has been investigated before to study holographic duals of condensed matter systems. (See, for instance, Refs. [24-26] and references therein.)

Indeed, one can show that the Einstein-Maxwell-Higgs system is reduced to the Einstein theory coupled to a massive vector field by choosing a wine-bottle potential and making the scalar field condensed. Then it is known that the Einsteinmassive vector model admits the Schrödinger space-time $[6,7,10]$. In other words, although the NR hybrid geometry Eq. (1) contains three parameters, the parameter $\alpha$ can be explained by the Einstein-Maxwell-Higgs system. However, from our preliminary analysis, the rest of the parameters $\beta$ and $\gamma$ cannot be explained no matter how the scalar potential is chosen. Then a question is how we can take account of $\beta$ and $\gamma$ without adding any physical degrees of freedom to the bulk, or whether there exists a bulk theory to support the NR hybrid geometry as a solution.

A clue to answer this question is in the origin of $\beta$ and $\gamma$. As seen from the parameter identification, these parameters come from $\bar{m}$ contained in the flow equation. Since a choice of flow equation fixes how to perform course-graining of operators, a parameter in a flow equation is not a physical parameter in the theory. This implies that $\beta$ and $\gamma$ should be regarded as gauge degrees of freedom. (This is also suggested by the expectation value of the spin-1 field.) For this reasoning, we repeat the same analysis by adding a gauge-fixing term for the abelian gauge symmetry as well as that for diffeomorphism to the Einstein-Maxwell-Higgs system, and it turns out that the Einstein-Maxwell-Higgs theory with a gravitational gaugefixing term admits the NR hybrid geometry with the general three parameters. We will verify this argument in the next section.

\section{THE DUAL BULK THEORY}

As argued in the previous section, let us consider an Einstein-Maxwell system minimally coupled to a charged scalar field with a certain potential accompanied with a gravitational gauge-fixing term

$$
\begin{aligned}
S= & \int d^{d+1} x \sqrt{|g|}\left[\frac{1}{2 \kappa^{2}}(R-2 \Lambda)-\frac{1}{4} g^{\mu \nu} g^{\rho \sigma} F_{\mu \rho} F_{\nu \sigma}\right. \\
& \left.-g^{\mu \nu} D_{\mu} \Phi^{*} D_{\nu} \Phi-V\left(|\Phi|^{2}\right)-\frac{1}{2 \xi}\left(g^{--}\right)^{2}\right] .
\end{aligned}
$$

The first term is the Einstein-Hilbert term in the Einstein frame with a negative cosmological constant $\Lambda$. (In principle there is no way to distinguish between a cosmological constant and the expectation value of the potential physically. However, in a situation where NRCFT is obtained from a parent CFT by a certain deformation, it would be natural to think that the cosmological constant is unchanged under the deformation and the deviation of the vacuum energy of NRCFT from that of CFT is accounted for by a newly added term, namely the value of the scalar potential. Thus, we assume the same value of the cosmological constant of the AdS.) The second term is the Maxwell term with the canonical normalization. Note that the gauge coupling constant is normalized to be unity by employing the Weyl transformation. The third one is the kinetic term of a charged scalar field and the covariant derivative is defined as $D_{\mu} \Phi \equiv \partial_{\mu} \Phi+i A_{\mu} \Phi$, where the gauge charge of the scalar field was fixed to unity as expected from the boundary theory. The fourth term is the potential of the scalar field, which is supposed to have a minimum value away from the origin so that the gauge symmetry is spontaneously broken when the scalar field condensates at the potential minimum. The last term is a gauge fixing term for diffeomorphism, where $\xi$ is a gauge-fixing parameter. Remark that for our final result, it is important to fix diffeomorphism in the Lorentz noninvariant manner. For example, a Fierz-Pauli-like term $\frac{1}{2 \xi} g_{\mu \nu} g^{\mu \nu}$ or a gauge fixing term for the gauge symmetry such as $\frac{1}{2 \xi^{\prime}}\left(A_{-}\right)^{2}$ are not relevant to our final result.

Let us show that this system supports the nonrelativistic hybrid geometry Eq. (1) with the following configuration of the gauge field:

$$
A_{+}=\frac{a_{+}}{\tau^{2}}, \quad A_{-}=a_{-},
$$

where $a_{ \pm}$are real constants, which are related to the boundary theory by $a_{+}=-\frac{i \Delta G^{(0,1)}}{2 G^{(1,0)}}, a_{-}=-4 \bar{m} G^{(1,0)}$. To this end, it is sufficient to show that the metric Eq. (1) and the gauge field Eq. (8) satisfy the equations of motion.

The Einstein equation is given by

$$
G_{\mu \nu}+g_{\mu \nu} \Lambda=\kappa^{2} T_{\mu \nu}
$$

where $G_{\mu \nu}$ is the Einstein tensor and $T_{\mu \nu}$ is the stress-energy tensor for the matter fields computed as

$$
\begin{aligned}
T_{\mu \nu} & =T_{\mu \nu}^{A}+T_{\mu \nu}^{\Phi}+T_{\mu \nu}^{\mathrm{gf}}, \\
T_{\mu \nu}^{A} & =-\frac{1}{4} g_{\mu \nu} F_{\sigma \rho} F^{\sigma \rho}+g^{\rho \sigma} F_{\mu \rho} F_{\nu \sigma}, \\
T_{\mu \nu}^{\Phi} & =g_{\mu \nu}\left[-D^{\rho} \Phi^{*} D_{\rho} \Phi-V\left(|\Phi|^{2}\right)\right]+D_{\{\mu} \Phi^{*} D_{\nu\}} \Phi, \\
T_{\mu \nu}^{\mathrm{gf}} & =g_{\mu \nu}\left[-\frac{1}{2 \xi}\left(g^{--}\right)^{2}\right]+\frac{1}{\xi} \delta_{\mu}^{-} \delta_{\nu}^{-} g^{--} .
\end{aligned}
$$

Note that we have included the contribution coming from the gauge-fixing term in the stress-energy tensor.

With the convention $\Phi=r \mathrm{e}^{i \theta}$, and $W_{\mu}=A_{\mu}+\partial_{\mu} \theta$, which is a gauge invariant vector field, the equations of motion for the gauge field and the modulus field $r$ are given by

$$
\begin{aligned}
& \frac{1}{\sqrt{|g|}} \partial_{\mu}\left(\sqrt{|g|} F^{\mu \nu}\right)=2 r^{2} W^{\nu}, \\
& \frac{1}{\sqrt{|g|}} \partial_{\mu}\left(\sqrt{|g|} \partial^{\mu} r\right)=r\left[V^{\prime}\left(r^{2}\right)+W_{\mu} W^{\mu}\right] .
\end{aligned}
$$

For our special form Eq. (8) we desire to look for a solution with the modulus $r$ constant, which is realized by 
tuning a potential to satisfy $V^{\prime}\left(r^{2}\right)=-W_{\mu} W^{\mu}$. Interestingly, this solution is different from the position of the minimum of the scalar potential, as will be seen soon. Note that both in the stress-energy tensor and on the right hand side of the equation of motion $W_{\mu}$ behaves like a massive vector boson field with mass $2 r^{2}$. By using Eqs. (1) and (8), in the assumed parameter region Eq. (2), Eqs. (14) can be solved by $r=$ $\sqrt{\frac{(d-2) \alpha \gamma+d(\beta+1)^{2}}{\ell^{2}\left(\alpha \gamma+(1+\beta)^{2}\right)}}$ and $\theta=\left(-a_{-}+\frac{2 a_{+} \gamma(1+\beta)}{(d-2) \alpha \gamma+d(\beta+1)^{2}}\right) x^{-}$. Note that the value of $W_{\mu} W^{\mu}$ becomes constant for this solution.

For these configurations, the stress energy tensors are computed as

$$
\begin{aligned}
T_{\mu \nu}^{\Phi}= & g_{\mu \nu}\left\{\frac{\left(a_{+}\right)^{2} \gamma\left[(d-2)^{2} \alpha \gamma+d(d-4)(\beta+1)^{2}\right]}{\ell^{4}\left[\alpha \gamma+(\beta+1)^{2}\right]\left[(d-2) \alpha \gamma+d(\beta+1)^{2}\right]}\right. \\
& \left.-V\left(r^{2}\right)\right\}+2 r^{2}\left\{\left[\frac{a_{+} \gamma 2(\beta+1)}{(d-2) \alpha \gamma+d(\beta+1)^{2}}\right]^{2} \delta_{\nu}^{-} \delta_{\mu}^{-}\right. \\
& \left.+\frac{a_{+}^{2}}{\tau^{4}} \delta_{\mu}^{+} \delta_{v}^{+}+\frac{2}{\tau^{2}} \frac{a_{+}^{2} \gamma(\beta+1)}{(d-2) \alpha \gamma+d(\beta+1)^{2}} \delta_{\{\mu}^{-} \delta_{\nu\}}^{+}\right\}, \\
T_{\mu \nu}^{A}= & \left\{\frac{1}{2} g_{\mu \nu} \frac{\gamma}{\ell^{4}\left[\alpha \gamma+(1+\beta)^{2}\right]}+\delta_{\mu}^{+} \delta_{\nu}^{+} \frac{1}{\ell^{2} \tau^{4}}\right. \\
& \left.+\delta_{\mu}^{\tau} \delta_{v}^{\tau} \frac{-\gamma}{\tau^{2} \ell^{2}\left[\alpha \gamma+(1+\beta)^{2}\right]}\right\}\left(2 a_{+}\right)^{2}, \\
T_{\mu \nu}^{\mathrm{gf}}= & g_{\mu \nu}\left(-\frac{1}{2 \xi}\left\{\frac{\alpha}{\ell^{2}\left[\alpha \gamma+(1+\beta)^{2}\right]}\right\}^{2}\right) \\
& +\frac{1}{\xi} \delta_{\mu}^{-} \delta_{\nu}^{-} \frac{\alpha}{\ell^{2}\left[\alpha \gamma+(1+\beta)^{2}\right]} .
\end{aligned}
$$

The Einstein tensor with the contribution of the cosmological constant for the metric Eq. (1) is computed as

$$
\begin{aligned}
G_{\mu \nu}+g_{\mu \nu} \Lambda= & \frac{\alpha \gamma}{\alpha \gamma+(1+\beta)^{2}} \frac{g_{\mu \nu}}{\ell^{2}}+\frac{\delta_{\mu}^{\tau} \delta_{v}^{\tau}}{\tau^{2}} \frac{-2 \alpha \gamma}{\alpha \gamma+(1+\beta)^{2}} \\
& +\delta_{\mu}^{-} \delta_{v}^{-} \frac{\gamma\left[(d-2)(1+\beta)^{2}+d \alpha \gamma\right]}{\alpha \gamma+(1+\beta)^{2}} \\
& +\frac{\delta_{\mu}^{\{+} \delta_{v}^{-\}}}{\tau^{2}} \frac{2(1+\beta) \alpha \gamma}{\alpha \gamma+(1+\beta)^{2}} \\
& +\frac{\delta_{\mu}^{+} \delta_{v}^{+}}{\tau^{4}} \frac{\alpha\left[(d+2)(1+\beta)^{2}+d \alpha \gamma\right]}{\alpha \gamma+(1+\beta)^{2}}
\end{aligned}
$$

Plugging these into the Einstein equation, we obtain five equations to be satisfied from the components $(\mu, v)=$ $(i, j),(+,+),(+,-),(-,-),(\tau, \tau)$. The equation for the $(+,-)$ component is satisfied if $a_{+}^{2}=\frac{\ell^{2} \alpha}{2 \kappa^{2}}$, thus the gauge flux does not vanish, as expected. Then the equations for the $(+,+)$ and $(\tau, \tau)$ components are automatically satisfied. The equation for the $(-,-)$ component fixes the parameter in the gauge-fixing term as

$$
\frac{1}{\xi}=\frac{d(d-2)\left[\alpha \gamma+(1+\beta)^{2}\right]^{2}}{(d-2) \alpha \gamma+d(1+\beta)^{2}} \frac{\gamma \ell^{2}}{\alpha \kappa^{2}} .
$$

The equation for the $(i, j)$ component fixes the potential at $r^{2}$ as $V\left(r^{2}\right)=-\frac{2 a_{+}^{2} \gamma}{\Delta^{2}\left[\alpha \gamma+(1+\beta)^{2}\right]}$. This completes the verification of our claim. Note that the flow field determines the Newton constant $\kappa^{2}$ as $\kappa^{2}=\frac{G^{(0,2)}(\overrightarrow{0})}{2\left[i G^{(0,1)}(\overrightarrow{0})\right]^{2}}$.
TABLE I. An anticipated correspondence between the bulk dynamical fields and the boundary operators.

\begin{tabular}{lclc}
\hline \hline \multicolumn{1}{c}{ Operators in NRCFT } & & \multicolumn{2}{c}{ Bulk dynamical fields } \\
\hline Flowed primary operator & $\sigma$ & Charged scalar field & $\Phi$ \\
Gauge field operator & $\hat{A}_{\mu}$ & Gauge field & $A_{\mu}$ \\
Metric operator & $\hat{g}_{\mu \nu}$ & Metric tensor & $g_{\mu \nu}$ \\
\hline \hline
\end{tabular}

We comment that the Einstein-Maxwell-Higgs system reduces to the Einstein theory with a massive vector field by choosing the potential appropriately by the Higgs mechanism, where the mass of the vector field is $2 r^{2}$. This means that the Einstein-massive vector theory with the gauge-fixing term for diffeomorphism also supports the NR hybrid geometry as a solution.

\section{DISCUSSION}

In this paper we have considered the dual gravitational theory of a general NRCFT and proposed that the theory is the Einstein theory minimally coupled to an abelian gauge field and a Higgs field with a gravitational gauge-fixing term, by confirming explicitly that the theory admits the NR hybrid geometry.

In spite of our explicit verification, we do not fully understand the role of the gravitational gauge-fixing term in the context of holography. It seems that only zero or nonzero are meaningful about the parameter of the gauge-fixing term and induce an important effect to solve the equations of motion. This result may indicate that a particular choice of smearing would correspond to a particular gauge choice in the bulk. It is significant to elaborate this point in detail.

It is also interesting to test our proposal by computing correlation functions for both sides and comparing them based on the dictionary in Table I. An advantage of the flow equation method is that one can perform explicit computations by using traditional techniques of quantum field theory such as the $1 / N$ expansion, which enables one to verify his/her proposal in an analytic way.

\section{ACKNOWLEDGMENTS}

The authors thank Shigeki Sugimoto for his valuable comment and discussion during the YITP-W-19-10 on "Strings and Fields 2019." This work is supported in part by the Grant-in-Aid of the Japanese Ministry of Education, Sciences and Technology, Sports and Culture (MEXT) for Scientific Research (Grants No. JP16H03978, No. JP18H01214, No. JP19K03847), by a priority issue (Elucidation of the fundamental laws and evolution of the universe) to be tackled by using Post "K" Computer, by Joint Institute for Computational Fundamental Science, by the Supporting Program for Interaction-based Initiative Team Studies (SPIRITS) from Kyoto University, by the JSPS Japan-Russia Research Cooperative Program, and by the Hungarian Scientific Research Fund NKFIH (under Grant No. K116505). 
[1] G. 't Hooft, Conf. Proc. C930308, 284 (1993).

[2] L. Susskind, J. Math. Phys. 36, 6377 (1995).

[3] J. M. Maldacena, Adv. Theor. Math. Phys. 2, 231 (1998).

[4] E. Witten, Adv. Theor. Math. Phys. 2, 253 (1998).

[5] S. S. Gubser, I. R. Klebanov, and A. M. Polyakov, Phys. Lett. B 428, 105 (1998).

[6] D. T. Son, Phys. Rev. D 78, 046003 (2008).

[7] K. Balasubramanian and J. McGreevy, Phys. Rev. Lett. 101, 061601 (2008).

[8] C. Duval, M. Hassaine, and P. A. Horvathy, Ann. Phys. 324, 1158 (2009).

[9] S. Kachru, X. Liu, and M. Mulligan, Phys. Rev. D 78, 106005 (2008).

[10] M. Taylor, arXiv:0812.0530.

[11] T. Banks, M. R. Douglas, G. T. Horowitz, and E. J. Martinec, arXiv:hep-th/9808016.

[12] S. Aoki, K. Kikuchi, and T. Onogi, Prog. Theor. Exp. Phys. 2015, 101B01 (2015).

[13] S. Aoki, J. Balog, T. Onogi, and P. Weisz, Prog. Theor. Exp. Phys. 2016, 083 B04 (2016).

[14] S. Aoki, J. Balog, T. Onogi, and P. Weisz, Prog. Theor. Exp. Phys. 2017, 043B01 (2017).
[15] S. Aoki and S. Yokoyama, Prog. Theor. Exp. Phys. 2018, 031B01 (2018).

[16] M. Albanese et al. (APE Collaboration), Phys. Lett. B 192, 163 (1987).

[17] R. Narayanan and H. Neuberger, J. High Energy Phys. 03 (2006) 064.

[18] S. Aoki and S. Yokoyama, Nucl. Phys. B 933, 262 (2018).

[19] S. Aoki, J. Balog, and S. Yokoyama, Prog. Theor. Exp. Phys. 2019, 043 (2019).

[20] S. Aoki, S. Yokoyama, and K. Yoshida, Phys. Rev. D 99, 126002 (2019)

[21] T. Banks and N. Seiberg, Phys. Rev. D 83, 084019 (2011).

[22] D. Harlow and H. Ooguri, arXiv:1810.05338

[23] K. Balasubramanian and J. McGreevy, J. High Energy Phys. 01 (2011) 137.

[24] J. McGreevy, Adv. High Energy Phys. 2010, 723105 (2010).

[25] G. T. Horowitz, Lect. Notes Phys. 828, 313 (2011).

[26] N. Iqbal, H. Liu, and M. Mezei, in Proceedings of the TASI2010, String Theory and Its Applications (World Scientific, Singapore, 2011), pp. 707-815. 